Thomas W. Nicolai, Kai Nagel

\title{
High resolution accessibility computations
}

Chapter in book | Submitted manuscript (Preprint)

This version is available at https://doi.org/10.14279/depositonce-7727

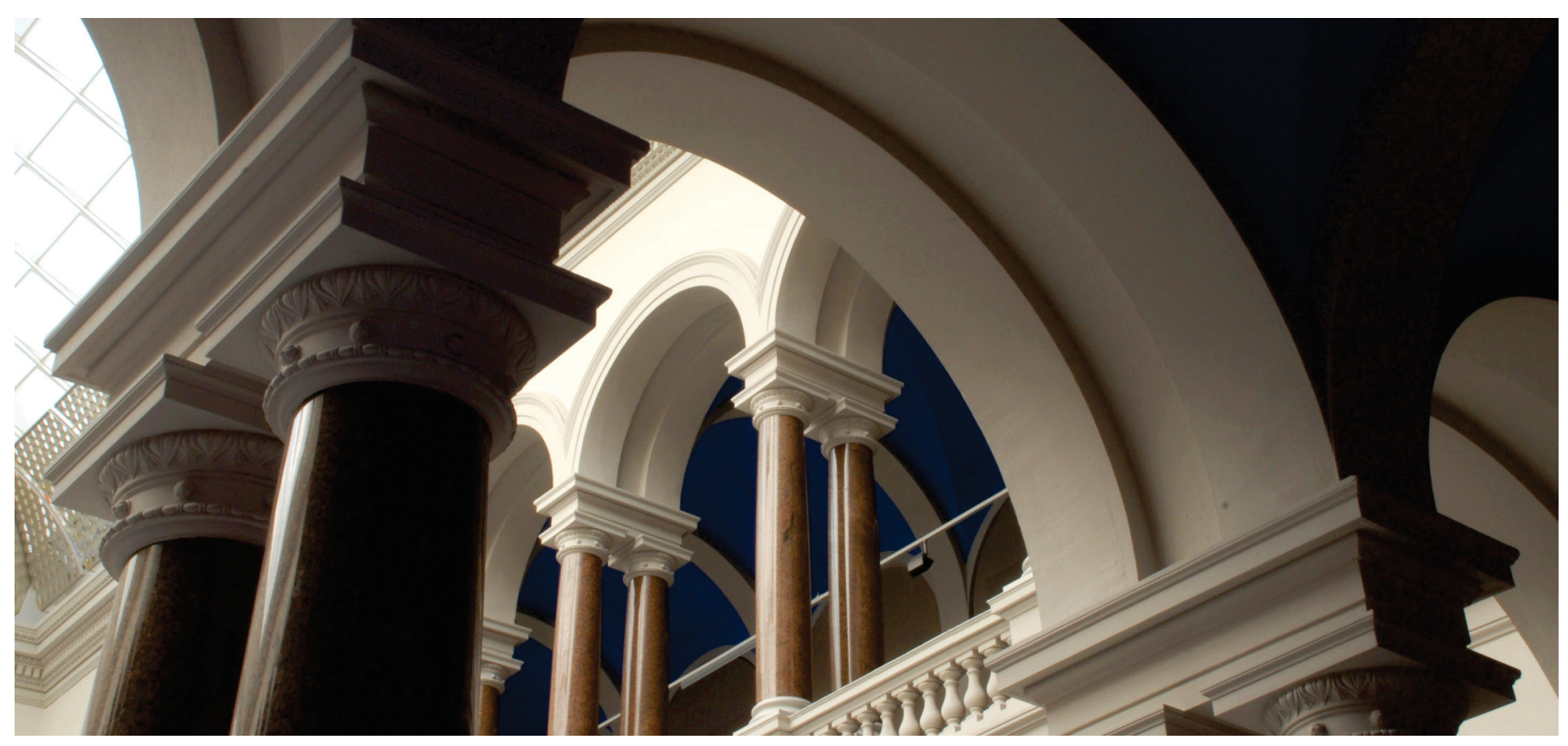

This is a draft chapter / article. The final version is available in "Accessibility and Spatial Interaction" edited by Condeço-Melhorado, A., Reggiani, A., Gutiérrez, J., published in 2014, Edward Elgar Publishing Ltd http://dx.doi.org/10.4337/9781782540731.00010

The material cannot be used for any other purpose without further permission of the publisher, and is for private use only.

Nicolai, T. W.; Nagel, K. (2014). High resolution accessibility computations. Accessibility and Spatial Interaction, 62-91. https://doi.org/10.4337/9781782540731.00010

\section{Terms of Use}

Copyright applies. A non-exclusive, non-transferable and limited right to use is granted. This document is intended solely for 


\title{
High resolution accessibility computations
}

\author{
Thomas W. Nicolai, Kai Nagel \\ Transport Systems Planning and Transport Telematics \\ Berlin Institute of Technology (TU Berlin) \\ Correspondence address: nagel@vsp.tu-berlin.de
}

July 31, 2013

\section{Introduction}

Researchers assert that accessibility has a measurable impact in the real world: Hansen (1959) shows that areas which have more access to opportunities have a greater growth potential in residential development. Moeckel (2006) asserts that the principal idea of Hansen's approach is also true for businesses. In other words: Locations with easier access to other locations are more attractive compared to otherwise similar locations with less access.

Many quantitative indicators can be used for accessibility (e.g. Geurs and Ritsema van Eck, 2001: Geurs and van Wee, 2004). Some examples are the distance to the next shopping area, travel time (e.g. Vandenbulcke et al., 2009) or distance (e.g. Borzacchiello et al., 2010) to the next railway station, or the number of opportunities (e.g. workplaces, places to shop) within, say, 1 kilometer. Accessibility can be seen as the result of the following four components (Geurs and Ritsema van Eck, 2001; Geurs and van Wee, 2004):

1. A land-use component that deals with the number and spatial distribution of opportunities.

2. A transport component, which describes the effort to travel from a given origin to a given destination.

3. A temporal component, which considers the availability of activities at different times-ofday, e.g. in the morning peak hours.

4. An individual component that addresses the different needs and opportunities of different socio-economic groups, e.g. different income groups.

Accordingly, accessibility measures can concentrate on one or several of these components (Geurs and Ritsema van Eck, 2001):

1. For example, the infrastructure-based approach is based on the performance of the transport system. An example would be the average speed by mode at certain locations.

2. The activity-based measurement deals with the distribution of possible activity locations in space and time. An example would be the number of shopping locations or workplaces within a certain distance. Alternatively, one could look at the number of shopping locations or workplaces within a certain travel time, which would combine the infrastructure-based with the activity-based approach.

3. A utility-based measurement of accessibility reflects the (economic) benefits, as the maximum expected utility, that someone gains from access to spatially distributed opportunities 
(Geurs and Ritsema van Eck, 2001; de Jong et al., 2007). The typical example is the logsum term, discussed below.

Normally, accessibilities are attached to locations $i$. That is, one can say that "location $i$ is very accessible", i.e. it is easy to reach, presumably from many different locations. Alternatively, one can say that "location $i$ has good accessibility to certain services", meaning that one is, starting at location $i$, able to get to many destinations. The present paper will concentrate on the second variant, "outgoing" accessibiity; the question in how far this is interchangeable with "incoming" accessibility will be addressed towards the end.

Furthermore, it will be assumed that our (quantitative) accessibility measure is of the mathematical form

$$
A_{i}=g\left(\sum_{j} a_{j} f\left(c_{i j}\right)\right)
$$

where the sum goes over all possible destinations (opportunities) $j, a_{j}$ is an indicator of the attractiveness of the opportunity, $c_{i j}$ is the generalized cost of travel to get from $i$ to $j, f(c)$ is an impedance function that typically decreases with increasing distance, and $g($.$) is an arbitrary,$ but typically monotonically increasing, function. That is, the accessibility at $i$ is computed from a weighted sum over all possible destinations, where the weight is the product of the destination's attractiveness and the ease to get there. This form subsumes most if not all of the measures discussed above $\left.\right|^{1}$ It includes the land-use component, since $a_{j}$ is larger than zero only at locations where the opportunity exists, and it includes the transport component via $c_{i j}$. It also includes the temporal component, since for practical computations the generalized cost of travel depends on the time-of-day, and it could depend on the individual person, by making attractiveness and impedance dependent on her demographic attributes. A collection of different quantitative accessibility measures is, for example, given by Martín and Reggiani (2007, Table 1).

Often, both the origin location $i$ and the destination locations $j$ are assumed to be relatively large zones. On the destination side, this is typically achieved by aggregating over all opportunities at zone $z$, and one obtains

$$
A_{i}=g\left(\sum_{j} a_{j} f\left(c_{i j}\right)\right) \approx g\left(\sum_{z} \sum_{j \in z} a_{j} f\left(c_{i z}\right)\right)=g\left(\sum_{z} a_{z} f\left(c_{i z}\right)\right),
$$

where $c_{i z}$ now is the generalized cost to travel from location $i$ to zone $z$, and $a_{z}$ is the aggregated attractiveness indicator of zone $z$. As one may note, there is the aggregation issue that $c_{i z}$ is not exactly the same as $c_{i j}$; this can cause problems for origins close to the zone, see below.

There are also issues on the origin side when using zones. For example, one could start the computation from a "typical" point in the zone, e.g. the zone centroid. Alternatively, one could start from all possible locations inside the zone and then average. The latter is prohibitively expensive to compute, as will become clear from this paper. However, using, as in the first option, just one point as typical for a zone is quite problematic since it is already intuitively clear that shifting such a point by, say, 500 meters can change accessibility by the walk mode quite significantly.

Finally, there is the issue that the aggregation of the destinations is problematic in the vicinity of the origin. For example, assume that you are in a zone without any opportunities, but close to a zone with many opportunities. Here, it makes no sense to assume that one has to travel to the centroid of the neighboring zone where then one finds all opportunities; rather, some opportunities may in fact be much closer. In that same vein, there has always been the issue of how to deal with opportunities that are in the same zone as the origin, sometimes called

\footnotetext{
${ }^{1}$ For example, the number of shopping opportunities within travel time $t^{*}$ can be obtained by summing over all shopping opportunities $j, a_{j}=1$ (all shopping locations equally attractive), $g(X)=X$, and$$
f\left(c_{i j}\right)=\left\{\begin{array}{cc}
1 & \text { if } t_{i j} \leq t^{*} \\
0 & \text { else }
\end{array}\right.
$$ 
the issue of self-potential (e.g. Frost and Spence, 1995, Fröhlich and Axhausen, 2004, CondeçoMelhorado et al. 2011): Should one assume that these opportunities have distance zero to the origin, i.e. that $c_{i j}=0$ for those destinations? Or should one assume a typical distance/travel time/generalized cost of travel depending on the average zone size? Or different such values for every zone? All of these somewhat arbitrary choices lead to different quantitative results, and because opportunities close to the origin have high weight, the differences can be quite important. In the present paper it will be shown that such choices do not need to be made, and instead accessibility can be computed with high enough resolution such that these issues do not arise.

In consequence, the present paper will, except for comparison purposes, assume that accessibility values are attached to points (also see Kwan, 1998) rather than zones. The accessibility computation for location $i$ will thus include, for each opportunity $j$, a generalized cost to reach the network, a generalized cost to travel on the network, and a generalized cost from the network to the opportunity. The network travel times will be based on the output of a dynamic traffic assignment, thus including congestion effects. This is similar in spirit to Kwan (1998), but goes beyond that contribution by (i) including the generalized cost of network access and egress, by (ii) using a much higher spatial resolution, and by (iii) using congested travel times from a dynamic assignment.

In fact, our whole perspective comes from simulation where we assign activity-based demand patterns dynamically onto the network (Balmer et al., 2009). Thus, the framework which evolves such patterns over many iterations, running a microscopic simulation of travel behavior in each iteration, is already there. The challenge is to generalize those behavioral patterns, for example to obtain the generalized cost of travel to opportunities that none of the persons starting at location $i$ ever visited. A second challenge derives from the SustainCity project (http://www. sustaincity.org), within which the present investigation was performed: Within that project, the travel model was expected to return accessibility values at parcel level. This will be discussed in somewhat more detail below.

Once $A_{i}$ is accepted as a measure relating to a single point, one can take this argument further and consider accessibility as a field, i.e. as continuously varying in space, $A(x, y)$, where $x$ and $y$ are the coordinates. As is common in many areas of science, such fields can be visualized by calculating the values on regular grid points, and then using an averaging plotting routine. Related approaches by other researchers fall into two classes:

- In the first class, accessibility is treated as a continuous field, but the accessibility measure is not computed on the congested network.

For example, Borzacchiello et al. (2010) use Euclidean distance. Bono and Gutiérrez (2011) do not use an accessibility measure similar to the ones discussed here, but measure the difficulty to reach the network.

- In the second class, accessibility is not treated as a continuous field; instead, the nodes of the transport network are used as origins. In return, many of these computations use the network, albeit often not a network with time-dependent congestion.

Many of these (e.g. Gutiérrez and Gómez, 1999; Gutiérrez et al., 2010, Condeço-Melhorado et al. 2011) then interpolate between these nodes. However, it is clear that at the microscopic level one can improve beyond such an approach: Accessibility between nodes should be lower than at nodes, and not in between the two values. Sometimes, it is also not fully clear which interpolation scheme was used.

Arguably most similar to our approach are Liu and Zhu (2004); Zhu and Liu (2004). The main difference is that their approach is embedded into a GIS tool whereas ours is attached to an activity-based dynamic traffic assignment. This makes their version (presumably) easier to use while ours (presumably) has the more realistic time-dependent congestion patterns. Also, their origins and destinations are once more zones or zone centroids, being subject to the issues discussed earlier. Finally, their papers do not present computing times, which means that it is impossible to judge if they have realized the same computational performance as we have. 
The goals of this paper are accordingly:

- Define an accessibility measure for each point of the region.

- Clarify how access to and egress from the transport network are handled.

- For grid-based computations, clarify the effect of different spatial resolutions.

- Consider computational performance issues.

- Perform illustrative sensitivity studies in a real-world setting including the effect of traffic congestion.

Sec. 2 describes in detail how this accessibility measurement is implemented. Sec. 3 describes a real-world scenario to which the approach was applied. Sec. 4 presents results of this application, in particular with respect to spatial resolution and computing times. The paper ends with a discussion and a conclusion.

\section{Methodology: high resolution accessibility}

\subsection{Accessibility indicator}

This paper will specifically look at accessibility indicators of the form

$$
A_{i}:=\ln \sum_{k} e^{V_{i k}}
$$

where $k$ goes over all possible destinations, and $V_{i k}$ is the disutility of travel in order to get from location $i$ to location $k$. Compared to Eq. (1), this thus uses $g()=.\ln (),. a_{j}=1, f\left(c_{i j}\right)=e^{-c_{i j}}$, and $-c_{i j}=V_{i j}$. This so-called logsum term has an econometric interpretation as the expected maximum utility (e.g. Ben-Akiva and Lerman, 1985).

The logsum term includes a land-use component that considers the number and distribution of opportunities, and a transport component that determines the effort to get there. It can also be interpreted as (the logarithm) of a weighted sum over opportunities, where each opportunity is weighted with $e^{V_{i k}}$, i.e. by the ease to get there. Clearly, in this formulation no other attributes of the opportunities are included.

It may be useful to recall the origins of Eq. (4). For this, assume that the full utility of location $k$, seen from $i$, is $U_{i k}=V_{b a s e}+V_{i k}+\epsilon_{i k}$, where $V_{b a s e}$ is a constant base utility for doing the activity at any location, $V_{i k}$ is the systematic (= observed) disutility to get there, and $\epsilon_{i k}$ is a random term which absorbs the randomness of the travel disutility, but more importantly the utility fluctuations around $V_{\text {base }}$ of doing the activity. Under the typical assumption that the $\epsilon_{i k}$ are independent and identically Gumbel-distributed random variables, the expectation value of $U_{i k}$ becomes

$$
E\left(U_{i}\right)=E\left(\max _{k} U_{i k}\right)=\text { Const }+\ln \sum_{k} e^{V_{i k}} \equiv \text { Const }+A_{i} .
$$

Const is an integration constant which can, in principle, be computed. It contains both the effect of the base utility, $V_{\text {base }}$, and some constants related to the Gumbel distribtion. Since it is the same for all locations, it is typically dropped. As a result, $A_{i}$ can become negative.

Eq. (4) sometimes includes a so-called scale parameter. See Sec. 5 for a discussion of this.

It is important to note that the sum will go over all opportunities $k$ separately. For example, if there are $N_{\ell}$ opportunities at a location, the term $e^{V_{i \ell}}$ will be added $N_{\ell}$ times. Clearly, this leads to the same result as

$$
\ln \sum_{\ell} N_{\ell} e^{V_{i \ell}}
$$


that is, multiple opportunities at the same location can be aggregated right away. The following investigation will instead use a method that aggregates also over close-by locations, reducing computing time even more. For the time being, it is best to assume that each opportunity is included separately.

Accessibilities in the present paper are computed based on a congested road network with time dependent travel times. This task is part of an attempt to couple a land use model, UrbanSim (Waddell, 2002; Miller et al., 2005: OPUS User Guide, 2011), with a transport model, MATSim (Balmer et al., 2005; Raney and Nagel, 2006; Balmer et al., 2009). In that configuration MATSim performs a traffic flow simulation based on the land-use and commuting patterns provided by UrbanSim. The inputs from UrbanSim consist of a list of persons, with references to households and, if applicable, to jobs. Both the households and the jobs refer, via intermediate steps, to locations, i.e. to zones or to parcels. Additionally, road network information is read by MATSim. The current version of MATSim4UrbanSim constructs simple home-work-home commuting plans from that information, which are then used as the initial demand for MATSim. MATSim relaxes that demand over typically 100 iterations, performing both a route and a departure time assigment. As a result MATSim possesses a congested road network with time dependent travel times. The feedback to UrbanSim currently consists of the following indicators: (i) zone-to-zone travel times and generalized travel costs; (ii) accessibility (as in the present paper) for each zone or each parcel, where parcels correspond to individual lots; (iii) each simulated person's travel time and generalized travel cost of the commute. A comprehensive description of the simulation and integration approach of MATSim and UrbanSim is given by Nicolai and Nagel (2012, pp. 21).

\subsection{Accessibility of location $i$}

\subsubsection{Overview}

In order to calculate the accessibility $A_{i}$, origin location $i$ and opportunity locations $k$ are assigned to a congested road network with time dependent travel times. For every given origin $i$ a so-called "least cost path tree" computation runs through the network and determines the best route, and thus the least negative travel utility $V_{i k}$, to each opportunity location $k$ by using the Dijkstra shortest path algorithm (Dijkstra, 1959). The best route from $i$ to $k$ depends on the given generalized cost such as link travel times or distances. Once the least cost path tree has explored all nodes, the resulting disutilities $V_{i k}$ for all opportunities are queried and the accessibility is calculated as stated in Eq. (4).

\subsubsection{Assignment of locations to the network}

Origin and opportunity locations do not necessarily lie on the network. Thus, the calculation of $V_{i k}$ includes the disutility of travel to overcome the gap between locations and the road network.

For origin locations $i$, the shortest distance to the network is either given by (i) the Euclidean distance to the nearest node or (ii) the orthogonal distance to the nearest link on the network. If the mapping of location $i$ is to a link, as in case (ii), $V_{i k}$ further includes the travel disutility to overcome the distance to the nearest node. The travel costs on the link are calculated by dividing the distance to the node by the travel speed of the considered transport mode, e.g. car (free speed or congested car travel times at a given time-of-day), bicycle, or walk.

For opportunity (= destination) locations $k$, the Euclidean distance to the nearest node is used to determine the shortest distance to the network.

\subsubsection{Disutility of travel}

As stated in Eq. (4), the computation of the accessibility for a given origin location $i$ contains a summation of the term $e^{V_{i k}}$ for all opportunity locations $k$. The determination of the disutility of travel, $V_{i k}$, consists of the following contributions, as depicted in Fig. 1: 


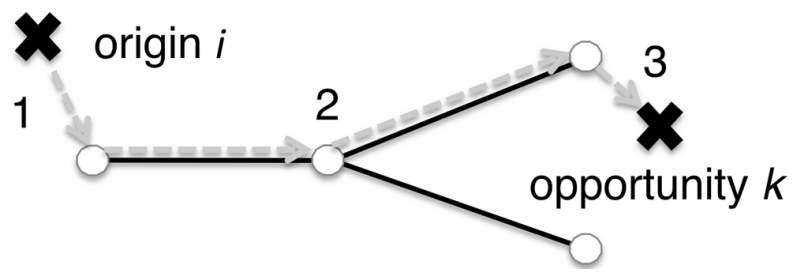

Figure 1: The composition of the travel disutility $V_{i k}$ consists of three parts: (1) the disutility to reach the network from $i,(2)$ the disutility on the network and (3) the disutility to reach opportunity $k$ from the network.

$$
V_{i k}:=\beta_{t t_{w l k}} \cdot t t_{w l k, g a p, i}+\beta_{t t_{\text {mode }}} \cdot t t_{\text {mode }}+\beta_{t t_{w l k}} \cdot t t_{w l k, g a p, k}
$$

where

- $t t_{w l k}$ is the travel time on foot.

- $t t_{\text {mode }}$ is the travel time according to the given transport mode. In the current implementation, transport modes are either car (free speed or congested), public transit, bicycle or walk.

- $t t_{w l k, g a p, i}$ is the travel time on foot to overcome the gap between the origin location $i$ and the road network.

- $t t_{w l k, g a p, k}$ is the travel time on foot to overcome the gap between the road network and the opportunity location $k$.

- $\beta_{t t_{\text {mode }}}$ and $\beta_{t t_{w l k}}$ are marginal utilities that convert travel times into utils. By default all marginal utilites are set to $-12 \mathrm{utils} / \mathrm{h}$. In MATSim terms, this is the sum of the marginal opportunity cost of time (typically $-6 \mathrm{utils} / \mathrm{h}$ ) and the marginal additional disutility of travel (typically another $-6 \mathrm{utils} / \mathrm{h}$ ).

The congested car speed comes from a dynamic traffic assignment, see Sec. 3.3, and thus reflects realistic congestion patterns. Public transit uses a so-called "matrix based public transit", which uses matrices of attributes such as travel time or distance between public transit stops (see http://matsim.org/matrixbasedptrouter). The travel times for traveling by bicycle or on foot are computed by taking the travel distance with a constant velocity of $15 \mathrm{~km} / \mathrm{h}$ (bicycle) or $5 \mathrm{~km} / \mathrm{h}$ (walk).

In all cases, the generalized cost of travel is computed according to the same principles as it is done in the dynamic traffic assignment; in the current implementation, also the utility weights are the same. One consequence of this is that the accessibility in fact refers to a certain timeof-day. Technically, the least cost path tree computation, anchored at the origin $i$, starts at a specific time-of-day, and then executes a time-dependent Dijkstra shortest path algorithm (Lefebvre and Balmer, 2007). Accessibility of the same location at a different time-of-day will usually be different, since congestion patterns are different.

\subsection{Spatial resolution}

When looking at high-resolution accessibility calculations, there are, in fact, two resolutions to consider: One that defines for how many origins $i$ the accessibility is to be computed. And a second one that defines to what level the opportunities $k$ are to be resolved.

Spatial resolution of the origin In the present implementation, the origin side can be calculated for two spatial units, cells or zones. Their spatial resolution determines the number of measuring points for which the accessibility will be computed: 


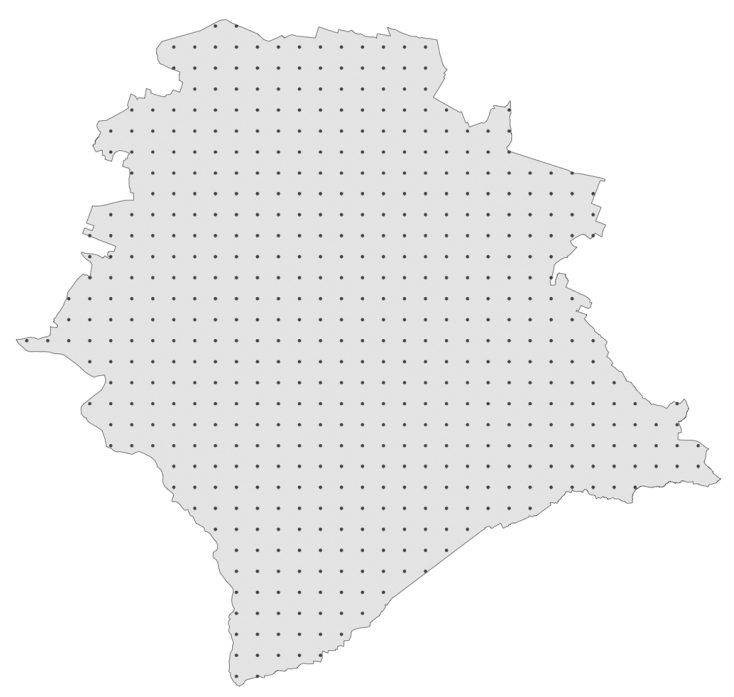

(a) Cell-based approach a the example of $400 \mathrm{~m} \times 400 \mathrm{~m}$ side length

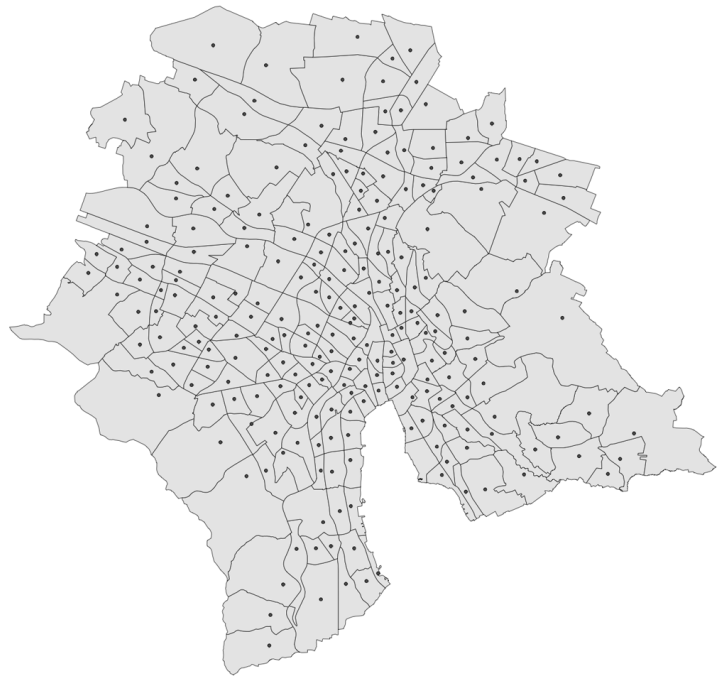

(b) Zone-based approach

Figure 2: The figures visualize the (a) cell- and (b) zone-based approach in accessibility calculation at the example of the city of Zurich (gray area). The origins or measuring points for accessibility calculation are determined as follows: The cell-based approach subdivides the study area in square cells of configurable size; here a side length of $400 \mathrm{~m} \times 400 \mathrm{~m}$ is used for visibility reasons. The cell centroids (dots) serve as origins. The zone-based approach is using zone centroids instead, which are determined by averaging all (UrbanSim) parcel coordinates that belong to a zone. The number of measuring points is given by the number of zones.

- Cell-based Approach: In this approach the study area is subdivided into square cells, where the resulting cell centroids serve as origins or measuring points for the accessibility calculation; see Fig. 2(a), The spatial resolution depends on the selected cell size, which is configurable.

- Zone-based Approach: This approach uses zone centroids as measuring points, see Fig. 2(b). The centroid coordinates can be obtained from a variety of definitions. In this paper, they are determined by averaging all parcel coordinates that belong to a zone. This corresponds to weighting each parcel equally; this may not be justified when, say, the number of residents or households varies strongly between parcels. The number of measuring points is defined by the number of zones.

In both cases, the accessibility computation is valid for the measuring point. In the grid-based approach it is then assumed that accessibility for in-between locations should be interpolated. In contrast, for the zone-based approach the accessibility of the measuring point is taken as representative for the whole zone. In consequence, the choice of the procedure of how to generate the measuring point for the zone-based approach has an influence on the results. There is no corresponding choice for the grid-based approach, removing one element of arbitrariness compared to the zone-based approach.

The following paragraphs concentrate on the cell-based approach. Nevertheless, the calculation procedure of the logsum term is the same for both approaches.

Spatial resolution of opportunities Opportunity locations such as work places are given by landuse. As stated earlier, they are attached to the nearest network node. The fact that they are attached to the nearest network node rather than the nearest network element (i.e. including links) is, in fact, the only approximation that the present paper makes with respect to the spatial resolution of opportunities. 


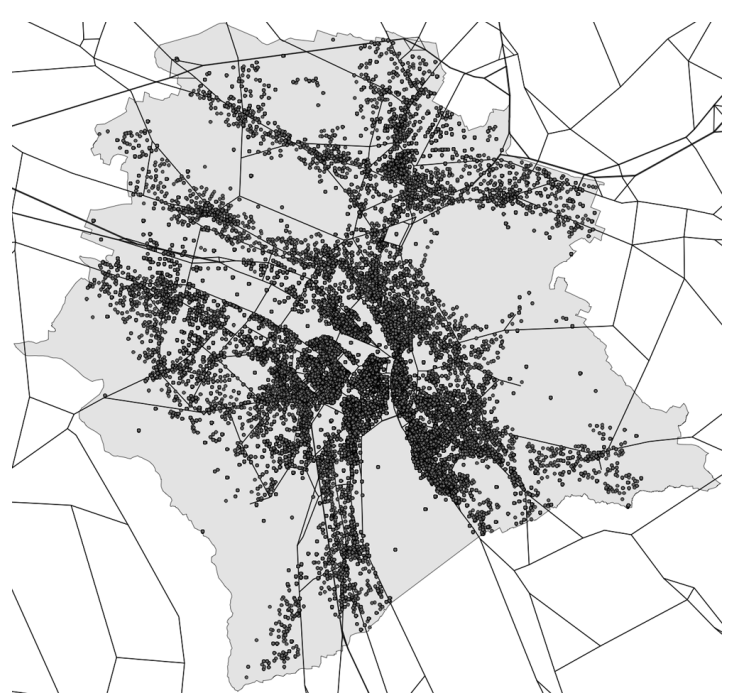

(a) Opportunities with parcel coordinates given by the land-use model

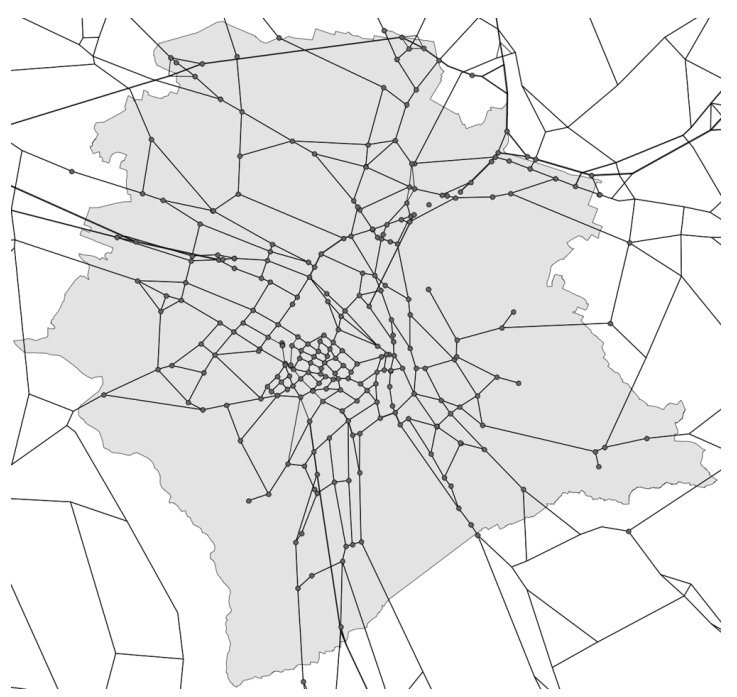

(b) Opportunities, aggregated on the nearest node on the road network

Figure 3: In (a) opportunity locations (dots) provided by the land-use model are at a disaggregated parcel level. The spatial resolution inside MATSim depends on the resolution of the road network, i.e. on the number of nodes and link lengths. Thus, opportunities are directly aggregated to their nearest node on the given road network as depicted in (b).

\subsection{Computational procedures}

Exploring the entire network by using the "least cost path tree" is a computationally expensive task. In order to accelerate the overall computing speed, the execution time of the "least cost path tree" is reduced by the following elements.

Origins For each origin location $i$, the nearest node on the road network is identified. Locations that share the same node have the same travel disutilities on the network. In this case the "least cost path tree" is executed only once and the calculated disutilities on the network are reused for all $i$ that are mapped on the same node. Only the calculation of the travel disutility to overcome the gap between location $i$ and the network is done individually.

This implies the approximation that all origins assigned to the same node use the same time-ofday as starting time for the time-dependent least cost path tree algorithm.

Opportunities It is, in fact, sufficient to sum over all opportunities $k$ attached to a node $j$ only once. For this, assume that the travel disutility $V_{i k}$ can be decomposed as

$$
V_{i k}=V_{i j}+V_{j k} \forall k \in j
$$

where the notation $k \in j$ shall refer to all opportunities $k$ attached to node $j$. Then

$$
\sum_{k \in j} e^{V_{i k}}=\sum_{k \in j} e^{\left(V_{i j}+V_{j k}\right)}=\sum_{k \in j} e^{V_{i j}} e^{V_{j k}}=e^{V_{i j}} \sum_{k \in j} e^{V_{j k}}=: e^{V_{i j}} \cdot O p p_{j}
$$

Thus, it is sufficient to compute $O p p_{j}$ once for every network node $j$, and from then on compute accessibilities by

$$
A_{i}=\ln \sum_{k} e^{V_{i k}}=\ln \sum_{j} e^{V_{i j}} \cdot O p p_{j} .
$$

This is an exact result. The only approximation was already done earlier; it is the assumption that all opportunities are reached through the nearest network node, rather than directly from the links. 


\section{Scenario: Zurich, Switzerland}

The above approach is applied to a real-world scenario. This is the city of Zurich, a parcel-based UrbanSim application that will be briefly discussed here. A full description is given by Schirmer et al. (2011); Schirmer (2010).

The Zurich application consists of 40'407 parcels, 336'291 inhabitants, and 316'703 jobs. In this paper the UrbanSim base year, 2000, is used to create the input for the MATSim runs. After that, UrbanSim is no longer needed for the present study.

\subsection{Population and Travel Demand}

In order to speed up computation times, MATSim considers a $10 \%$ random sample of the synthetic UrbanSim population, consisting of 33'629 agents. All MATSim agents have complete day plans with "home-to-work-to-home" activity chains. Work activities can be started between 7 and 9 o'clock, and have a typical duration of 8 hours. The home activity has a typical duration of 12 hours and no temporal restriction.

\subsection{Network and Adjustments}

A revised Swiss regional planning network (Vrtic et al., 2003; Chen et al., 2008) is used that includes major European transit corridors; see Fig. 4. The network consists of 24'180 nodes and 60'492 links, where each link is defined by an origin and a destination node, a length, a free speed car travel time, a flow capacity and a number of lanes. In addition each link obtains congested car travel times once the traffic flow simulation in MATSim is completed (see Nicolai and Nagel, 2012).

The flow and storage capacities of the road network are automatically adjusted based on the given population sampling rate used for the MATSim runs. This is done in order to preserve congestion effects when running MATSim at small samples. The flow capacity gives the maximum number of vehicles per time unit that can pass a link (Cetin et al., 2003). It is adjusted by a flow capacity factor, which is set to the same value as the given Population Sampling Rate. The storage capacity defines the maximum number of vehicles that can be on a link (Cetin et al. 2003). The corresponding storage capacity factor is defined as Population Sampling Rate/Heuristic Factor, where the Heuristic Factor $=$ Population Sampling Rate ${ }^{1 / 4}$. The Heuristic Factor aims to raise the storage capacity especially at low sampling rates to avoid network breakdowns caused by strong but spurious backlogs (Rieser and Nagel, 2008).

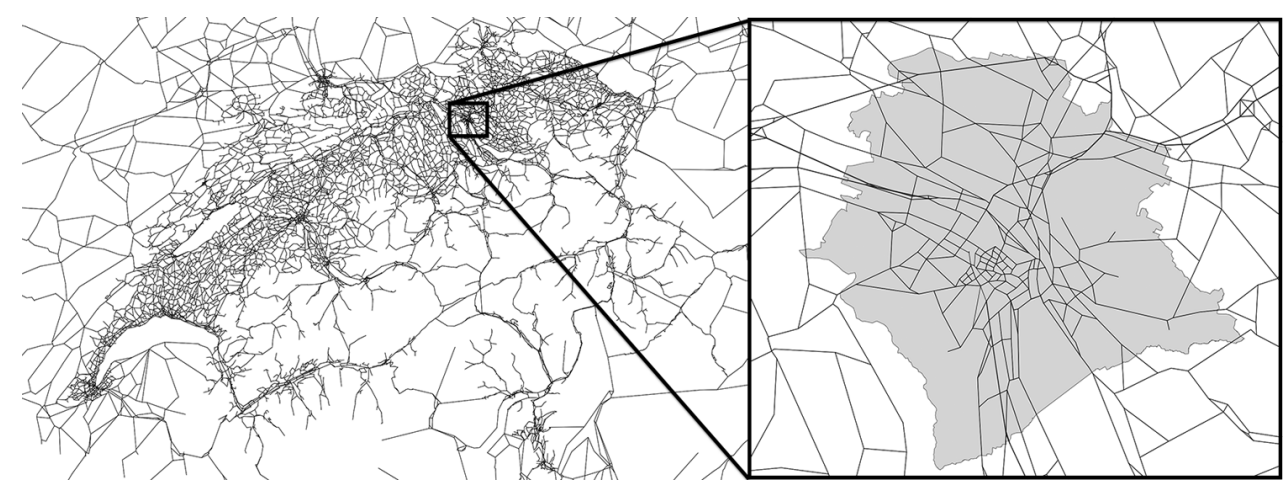

Figure 4: The Zurich case study network, area of Zurich (light gray) enlarged. 


\subsection{Traffic Simulation}

First, a base case MATSim run is performed by running the simulation for 1000 iterations. During the first 800 iterations $10 \%$ of the agents perform "time adaptation", which changes the departure times of an agent, and 10\% adapt their routes. The remaining agents switch between their plans. During the last 200 iterations time and route adaptations are switched off; thus, agents only switch between existing plans.

\section{Application}

This section will specifically look at the proposed high resolution accessibility approach at the example of work place accessibility. In particular the influence of the spatial resolution on the quality of the results and on computational performance are considered. In addition, as one example of a sensitivity test, the congested accessibility field is compared to uncongested accessibility as well as accessibility by bicycle and by walking.

All measurements are applied for the morning peak hour at 8am. At this time most travellers are commuting to work. Table 1 summarizes relevant parameter settings.

\begin{tabular}{|l|l|}
\hline \multicolumn{2}{c}{ Default Setting } \\
\hline \hline Resolution & $100 \mathrm{~m} \times 100 \mathrm{~m}$ \\
\hline Travel Cost & $\begin{array}{l}\text { congested car } \\
\text { travel times [minutes] }\end{array}$ \\
\hline$\beta_{t t_{c a r}}$ & $-12 \mathrm{utils} /$ hour \\
\hline$\beta_{t t_{w l k}}$ & $-12 \mathrm{utils} /$ hour \\
\hline \hline
\end{tabular}

Table 1: Default settings for the accessibility computation.

\subsection{Default setting}

Figure 5 depicts the accessibility outcome using the "Default Setting" as stated in Tab. 1. To improve interpretability, the road network is overlayed. The scale bar on the right hand side indicates the accessibility level. Good work place accessibility is indicated by white areas and poor accessibility is indicated by dark gray or black areas.

The plot exhibits very good work place accessibility in areas that provide a high density of opportunities and a well developed road network. These characteristics apply for the inner city, where the highest accessibility values are measured, and the areas along the major access roads from and to Zurich, visible as white or light gray corridors. In contrast, areas with less workplace accessibility have a gradient from dark gray to black. This for instance applies for the "Zürichberg" and the "Uetliberg", which are two undeveloped wooden hills located in the eastern and south-western part of Zurich. The several "islands of low accessibility" in the center of Zurich are due to localized congestion on those links: If there is strong congestion at the origin, then all opportunities $k$ incur a strongly negative $V_{i k}$ and thus make only a small contribution to the sum.

It should be noted that accessibility is now smooth in space, see Fig. 6. Figure 6(a) is the same as Fig. 5 but without the road network. One clearly sees how highly accessible areas trace the road network. The zone representation of the same data is shown in Fig. 6(b). Clearly, all additional spatial resolution within the zones is now gone. Also, with the zones approach the question in how far the transport network becomes visible in the accessibility plot hinges entirely on the question if the zones reflect the structure of the transport network or not. Such arbitrariness is removed with an approach that does not rely on zones. 


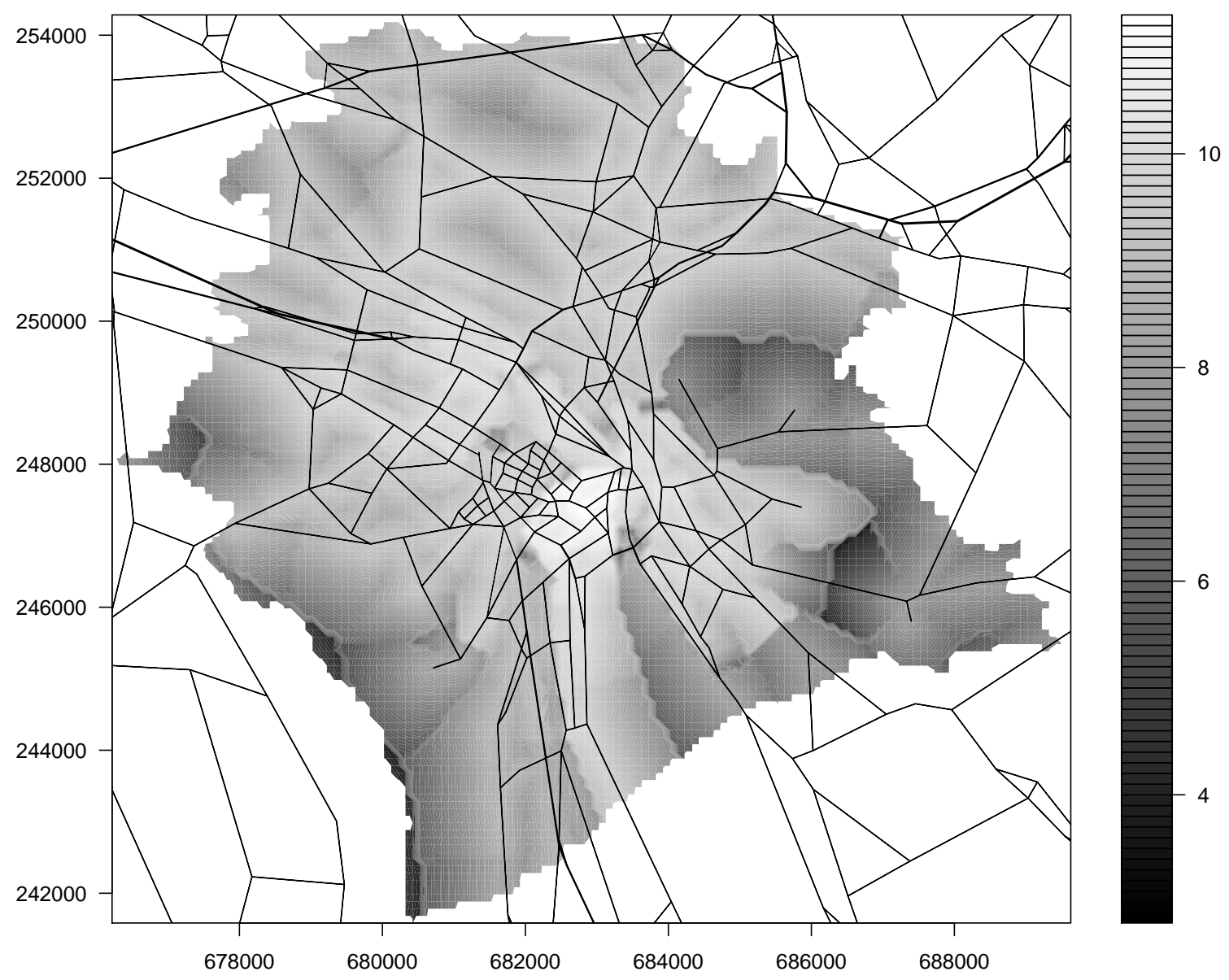

Figure 5: This depicts the outcome of the proposed accessibility measurement using the "Default Settings" as stated in Tab. 1.
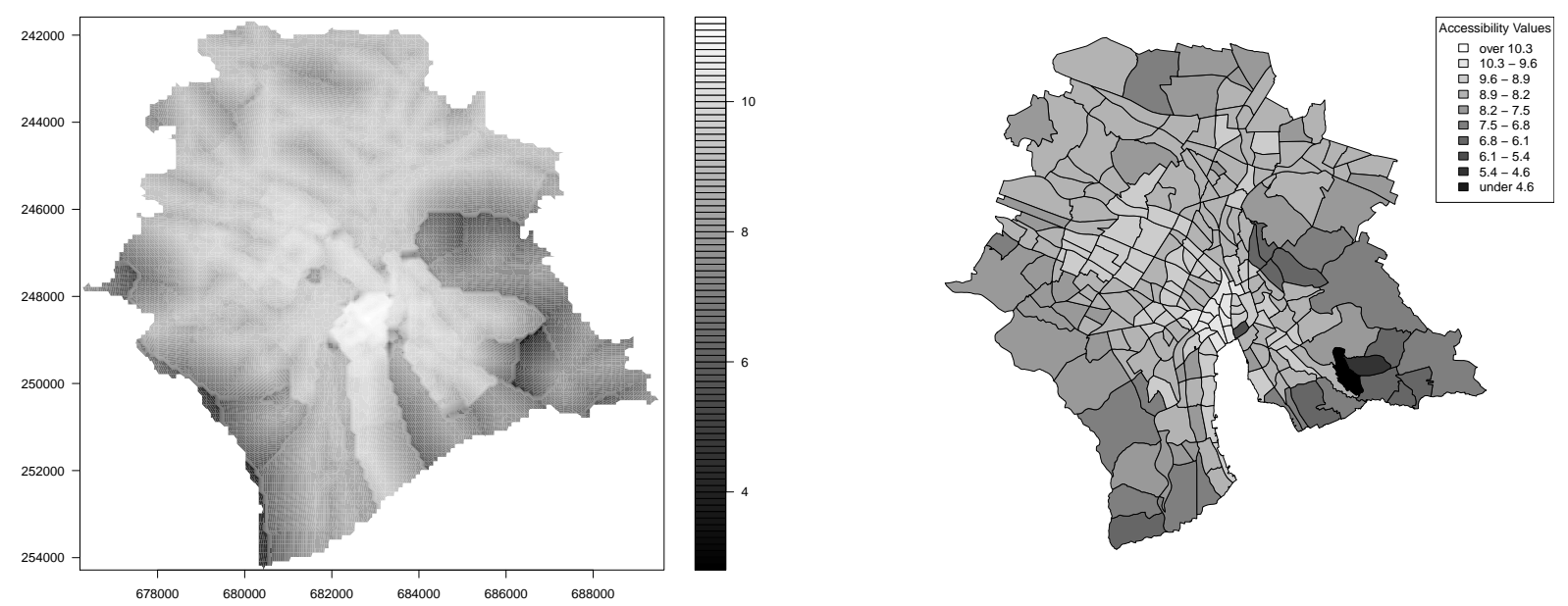

(a) The same as Fig. 5 but without showing the road network. (b) Accessibility based on the same congestion data as Fig. 5 but now based on zones as is traditionally done.

Figure 6: Alternative representations of accessibility based on the same congestion data as Fig. 5 . 


\subsection{Spatial resolution}

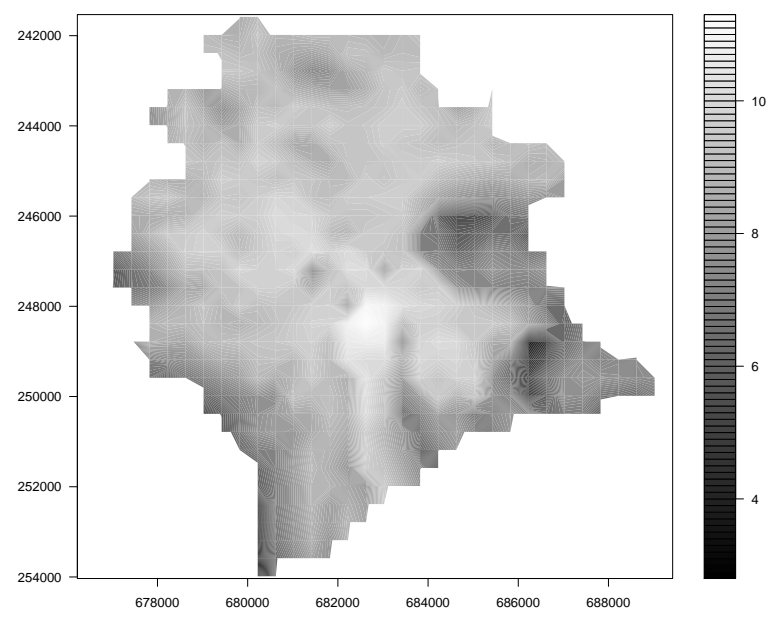

(a) Resolution $400 m \times 400 m$

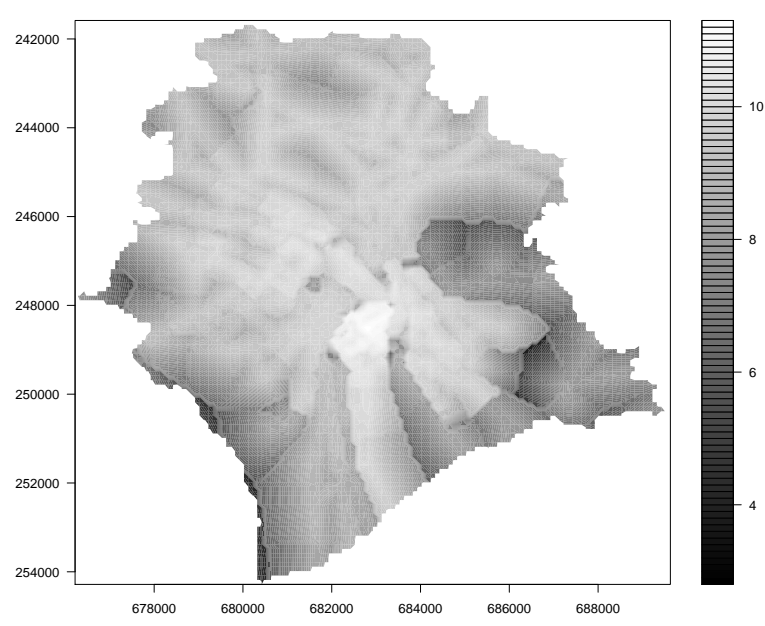

(c) Resolution $100 \mathrm{~m} \times 100 \mathrm{~m}$

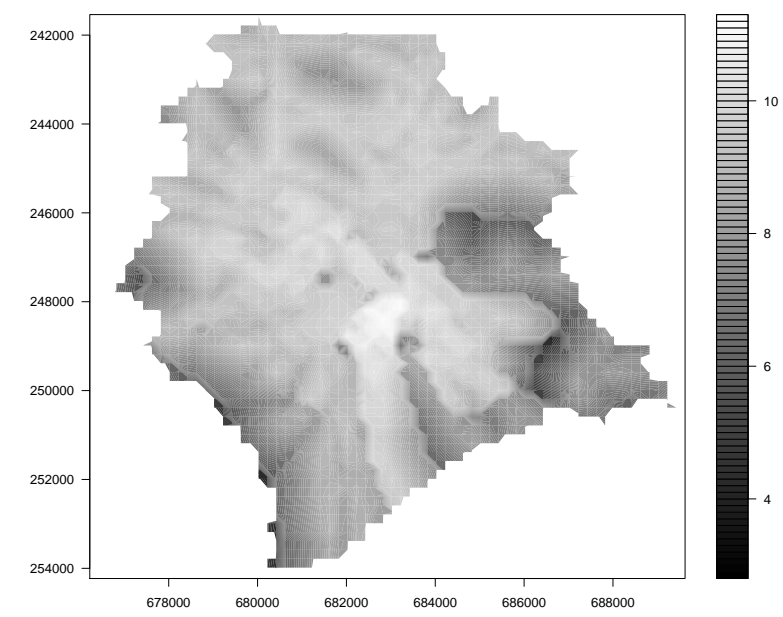

(b) Resolution $200 m \times 200 m$

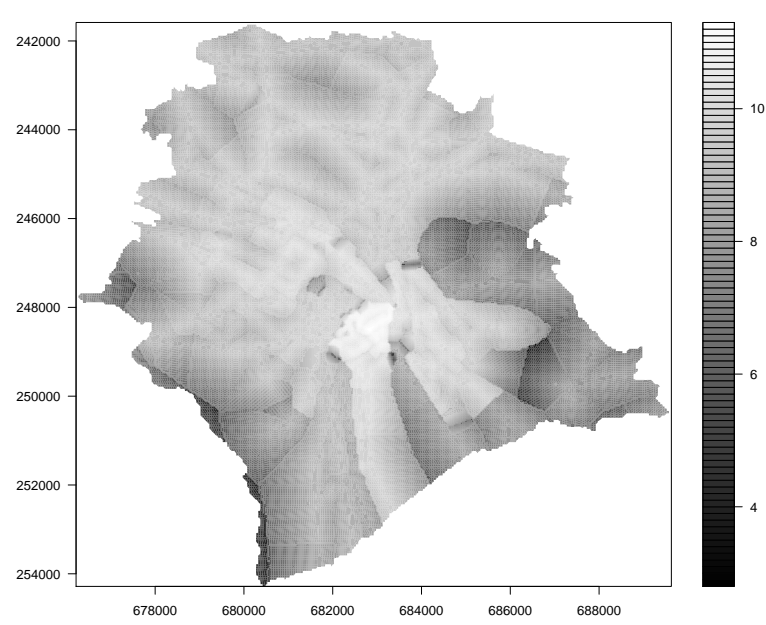

(d) Resolution $50 \mathrm{~m} \times 50 \mathrm{~m}$

Figure 7: Results of different resolutions. In ascending order from the top left to the bottom with the following cell sizes $400 m \times 400 m, 200 m \times 200 m, 100 m \times 100 m$ and $50 m \times 50 m$.

Figure 7 shows the outcome of the accessibility measurement at the following resolutions: $50 \mathrm{~m} \times$ $50 m, 100 m \times 100 m, 200 m \times 200 m$ and $400 m \times 400 m$. In terms of information gain it can be stated that the lowest resolution with $400 \mathrm{~m} \times 400 \mathrm{~m}$ provides a rather undifferentiated picture, whereas higher resolutions lead to more detailed measurements. In the $50 \mathrm{~m} \times 50 \mathrm{~m}$ resolution, even fine road structures in the city center are clearly visible. However, a significant increase in the level of detail can be observed up to the resolution of $100 \mathrm{~m} \times 100 \mathrm{~m}$. The higher resolution $(50 m \times 50 m)$ looks smoother and sharper, but does not offer noticeable gains.

\subsection{Computing times}

In Sec. 4.2, the resolution of two successive plots is doubled. This corresponds to a quadrupling of the measuring points. However, this increase is not reflected in the computing times, see Tab. 2, due to the run time optimizations outlined in Sec. 2.4. Instead, there is no advantage in terms of computing speed for the traditional zone based accessibility measure despite the low 


\begin{tabular}{llll}
\hline Cell Resolution & Origins & Aggregated Opportunities & Computing Time [min] \\
\hline \hline $50 m \times 50 m$ & 36748 & 272 & $2-3$ \\
\hline $100 m \times 100 m$ & 9195 & 272 & 2 \\
\hline $200 m \times 200 m$ & 2292 & 272 & $\approx 2$ \\
\hline $400 m \times 400 m$ & 577 & 272 & $\approx 1$ \\
\hline \hline Zone Resolution & Origins & Aggregated Opportunities & Computing Time [min] \\
\hline \hline Given by zones & 234 & 272 & $\approx 1$ \\
\hline \hline
\end{tabular}

Table 2: This table lists the computation times to measure accessibility at different resolutions and the zone level. All measurements are performed on a Mac Book Pro with an Intel Core 2 Duo $2.5 \mathrm{GHz}$ processor and $4 \mathrm{~GB}$ of memory. Currently $1 \mathrm{CPU}$ core is used to execute the accessibility computations.

resolution.

Computing times could be further improved by using multiple threads: Since the computations for different origins are independent, they could be distributed between all available CPU cores.

\subsection{Mode}

The results of congested vs. uncongested car and of using bicycle or walk mode are shown in Fig. 8. The first two plots are illustrating how congestion significantly reduces accessibility.

The third and fourth plot illustrate accessibility by bicycle and by walking, respectively. Congestion has no effect on these modes. Both plots show that spatial proximity to opportunities has a strong influence on the results. Locations away from the city center and the commercial areas in the northeast and western part of Zurich have a rapidly decreasing accessibility. As seems plausible, near the city center the bicycle provides similar accessibility as the car under congested conditions. Farther away from the center, the car gains ground, even under congested conditions.

One might speculate that Fig. 8(a) is what people who plan to use a car expect but Fig. 8(b) is what they actually get. And what they actually get is not much different from what one gets by using a bicycle (Fig. 8(c) . In contrast, walk is truly much worse (Fig. 8(d)].

\section{Discussion}

The unit of the accessibility measurement The logsum term, Eq. (4), sometimes contains a scale parameter, i.e. it reads $(1 / \mu) \ln \sum_{k} \exp \left(\mu \tilde{V}_{i k}\right)(*)$. When the utility function is estimated, Eq. (4) is the correct form (also see Train, 2003). Clearly, the user can decompose the estimated utility function $V_{i k}$ as $V_{i k}=\mu \tilde{V}_{i k}$, in which case Eq. (4) needs to be replaced by Eq. (*) in order to obtain the accessibility in the rescaled units. This may, for example, be useful if the utility function is to be scaled to monetary units, and accessibility is to be expressed in those same monetary units. Since $\mu \tilde{V}_{i k}$ is the same as $V_{i k}$, such re-scaling does not change the structure of the plots; however, all accessibility values will be multiplied by $1 / \mu$.

Spatial resolution Most plots in this paper are based on a grid resolution of $100 m \times 100 m$, and the road network as shown in some of the figures. As it was argued, higher spatial resolutions with the same road network do not lead to discernible improvements. In contrast, a higher resolution road network would clearly make a difference (also see next). Using a higher resolution road network is feasible - the computational cost scales roughly in the number of links since the worst case complexity of the Dijkstra tree computation is roughly linear in the number of links for planar graphs. In the present situation, it was decided to stick with the lower resolution network. One reason was that we wanted to investigate how our approach would perform in 


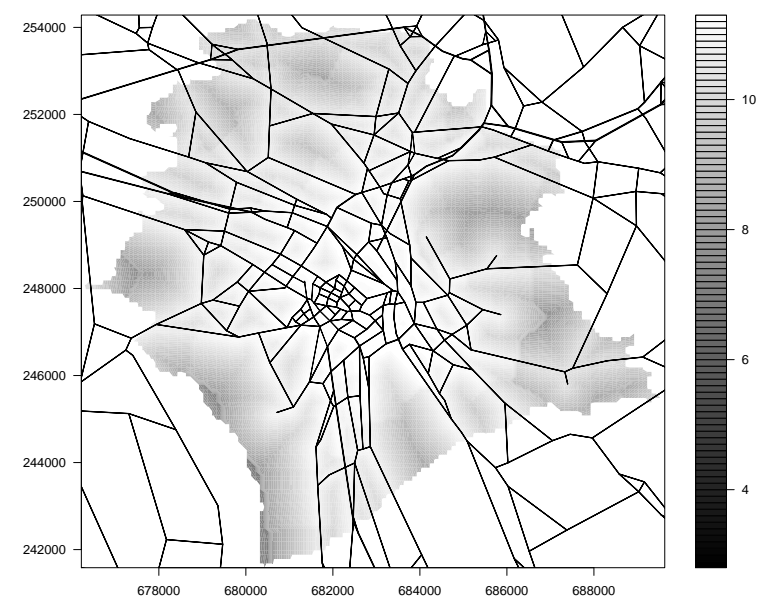

(a) Free speed car travel times in accessibility computation

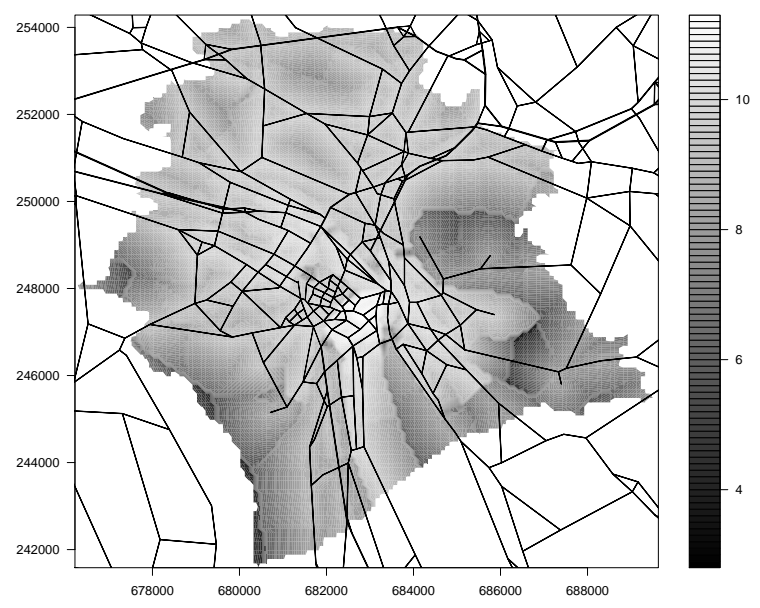

(b) Congested car travel times in accessibility computation (reference setting)

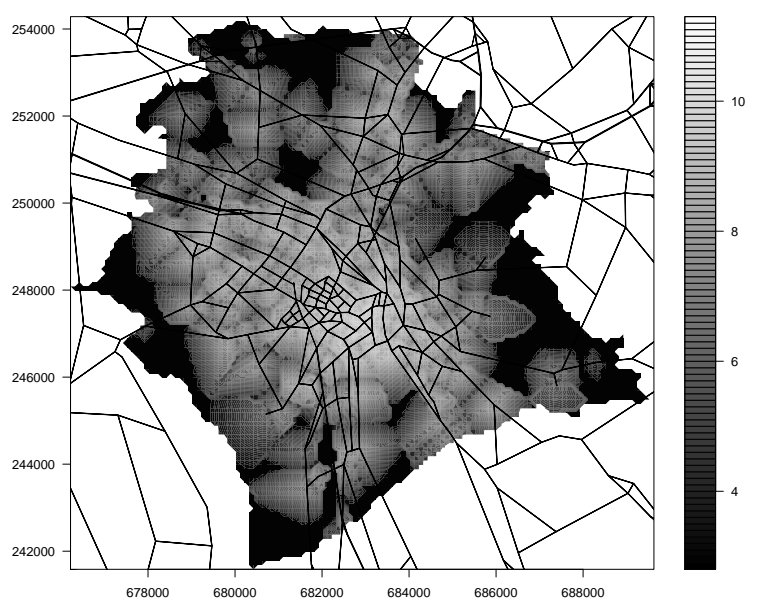

(d) Walk travel times in accessibility computation

(c) Bicycle travel times in accessibility computation

Figure 8: These plots visualize the influence of different transport modes on the accessibility computation.

such a situation. Another reason was that there was no higher resolution network together with a calibrated scenario available.

Connection to the transport network In the present investigation, origins are connected by the walk mode to the nearest network element (node or link), and destinations are connected by the walk mode to the nearest network node. Neither of these assumptions is always correct. For example, in the Zurich case there are additional roads beyond those that are used for the computation, and thus it is quite plausible that both for the car and for the bicycle modes, access to the network is much faster than assumed in the plots. This would reduce the "accessibility deprivation" that is currently visible (Fig. 5) in areas far away from the network. However, not all areas are in fact covered by such a secondary network, which makes it difficult to come up with a general specification for such areas, and which is the reason why the "walk" assumption was made.

Another issue is that origins are attached to the nearest network element, rather than to the network element that offers the highest level of accessibility. This sometimes causes seemingly sharp accessibility changes far away from the transport network, for example around the co- 
ordinates $(685000,249500)$ in Fig. 5. It might seem plausible to give origins a choice to which network element they want to attach, rather than forcing their attachment by such an arbitrary algorithm. On the other hand, in reality parcels are attached to the transport network by specific links, and in consequence one should not invent better algorithms but use better data. Also, the area around the above mentioned coordinates is, in fact, a communal forest, and thus the issue is, albeit not visually pleasing, of little practical relevance.

It is important to note that both issues are significantly reduced when moving to a higher resolution network, since any kind of approximation about how to reach the transport network will then have a smaller impact.

Directionality The introduction differentiated between "accessibility of a location to other locations that provide services" and "accessibility of a location providing services from other locations". The actual computations have concentrated on the first variant: a time-dependent shortest path tree was expanded from the origin location. The resulting measure could also be interpreted as the accessiblity of the location from other locations if travel effort was symmetric, i.e. if the effort to go from A to B was the same as the effort of going from B to A. Since the computations refer to a specific time-of-day, this will in general not be the case - for example, during the morning peak there is probably more congestion into the center than going out. That is, "origin accessibility" and "destination accessibility" at best approximate each other.

One could, however, modify the method described in the present paper to compute a high resolution destination accessibility. For this, one would start at the destination and expand a backwards shortest path tree, anchored at a specific arrival time.

Possible applications The concept of accessibility has always been used to analyse the "value" of locations. Moving it away from the zone and onto a point clarifies that it is really a "point" concept. As an important result, zones are no longer needed; accessibility can be computed for regions for which zones are not available; and all arbitrariness related to the definition of zones is removed. The approach also works equally well for slow modes such as walk and bicycle; only the network data needs to be more detailed.

Besides helping with location choice, the approach can also be used to systematically and automatically assess the access of locations to services, for example to schools, hospitals, groceries, and the like. For this the locations of these opportunities need to be known, but freely available data sources such as OpenStreetMap (openstreetmap.org) increasingly make such data available, or could be retrofitted even by a community effort if desired. Clearly, also the impedance function $f\left(c_{i k}\right)$ needs to be adapted in a meaningful way to the measure of interest.

\section{Conclusion}

Accessibility measures are, for a given origin, a weighted sum over possible destinations, where the weight typically is a decreasing function of generalized cost, possibly multiplied with an attractiveness indicator. In this paper, the econometric logsum term is used as an example. It can also be interpreted as a benefit that someone at a specific location derives from access to spatially distributed opportunities. It includes, as usual, a land-use component by considering the distribution of opportunities and a transport component by determining the effort to get there. Important results include:

- The approach is able to provide a spatially differentiated picture. Artefacts such as supposedly homogeneous accessibility within zones are removed.

- The computation of a cell-based accessibility measurement at high resolutions is computationally feasible. 
- For the present scenario and network a resolution finer than $100 \mathrm{~m} \times 100 \mathrm{~m}$ does not deliver noticeable additional gains.

- The effect of congestion is very clearly visible. The result also confirms that, in the urban core, accessibility by bicycle is similar to accessibility by car during congested peak hours. Outside the urban core, however, accessibility by bicycle is clearly worse than by car also during peak hours.

In contrast, accessibility when walking is definitely much worse than either by car of by biclye, and reaches levels comparable to accessibilities by bicycle or by car only in the inner center of the city.

\section{Acknowledgments}

This work has been funded in part by the EU within the Seventh Framework Programme (FP7) within the research project SustainCity. The code for the calculation and visualization of accessibilities is based on previous work by Johannes Illenberger. Michael Balmer has programmed the efficient and flexible "least cost path tree" algorithm that is at the heart of the accessibility computation. Michel Bierlaire provided access to the Zurich data. Michael Zilske was significantly involved in the runtime optimization of the accessibility calculation. Theresa Thunig implemented the interpolation routine.

\section{References}

Balmer, M., B. Raney, and K. Nagel. Adjustment of activity timing and duration in an agentbased traffic flow simulation. In H. Timmermans, ed., Progress in activity-based analysis, pp. 91-114. Elsevier, Oxford, UK, 2005.

Balmer, M., M. Rieser, K. Meister, D. Charypar, N. Lefebvre, K. Nagel, and K. Axhausen. MATSim-T: Architecture and simulation times. In A. Bazzan and F. Klügl, eds., Multi-Agent Systems for Traffic and Transportation, pp. 57-78. IGI Global, 2009.

Ben-Akiva, M. and S. R. Lerman. Discrete choice analysis. The MIT Press, Cambridge, MA, 1985.

Bono, F. and E. Gutiérrez. A network-based analysis of the impact of structural damage on urban accessibility following a disaster: the case of the seismically damaged Port Au Prince and Carrefour urban road networks. Journal of Transport Geography, 19(6), 1443, 2011. doi: 10.1016/j.jtrangeo.2011.08.002.

Borzacchiello, M. T., P. Nijkamp, and E. Koomen. Accessibility and urban development: A gridbased comparative statistical analysis of dutch cities. Environment and Planning B: Planning and Design, 37, 148, 2010. doi:10.1068/b34126.

Cetin, N., A. Burri, and K. Nagel. A large-scale agent-based traffic microsimulation based on queue model. In Proceedings of the Swiss Transport Research Conference (STRC). Monte Verita, CH, 2003. See www.strc.ch. Earlier version, with inferior performance values: Transportation Research Board Annual Meeting 2003 paper number 03-4272.

Chen, Y., M. Rieser, D. Grether, and K. Nagel. Improving a large-scale agent-based simulation scenario. VSP Working Paper 08-15, TU Berlin, Transport Systems Planning and Transport Telematics, 2008. See www.vsp.tu-berlin.de/publications.

Condeço-Melhorado, A., J. Gutiérrez, and J. C. García-Palomares. Spatial impacts of road pricing: Accessibility, regional spillovers and territorial cohesion. Transportation Research Part A: Policy and Practice, 45(3), 185, 2011. doi:http://dx.doi.org/10.1016/j.tra.2010.12.003. 
de Jong, G., A. Daly, M. Pieters, and T. van der Hoorn. The logsum as an evaluation measure: Review of the literature and new results. Transportation Research Part A: Policy and Practice, 41(9), 874, 2007. doi:10.1016/j.tra.2006.10.002.

Dijkstra, E. A note on two problems in connexion with graphs. Numerische Mathematik, 1, 269, 1959.

Fröhlich, P. and K. W. Axhausen. Sensitivity of accessibility measurements to the underlying transport network model. IVT Working paper 245, Institute for Transport Planning and Systems, ETH Zurich, Zurich, Switzerland, 2004.

Frost, M. E. and N. A. Spence. The rediscovery of accessibility and economic potential: the critical issue of self-potential. Environment and Planning A, 27(11), 1833, 1995.

Geurs, K. and J. Ritsema van Eck. Accessibility measures: review and applications. Tech. rep., National Institut of Public Health and the Environment, RIVM, P.O. Box 1, 3720 BA Bilthoven, 2001.

Geurs, K. T. and B. van Wee. Accessibility evaluation of land-use and transport strategies: review and research directions. Journal of Transport Geography, 12, 127 , 2004.

Gutiérrez, J., A. Condeço-Melhorado, and J. C. Martín. Using accessibility indicators and \{GIS\} to assess spatial spillovers of transport infrastructure investment. Journal of Transport Geography, 18(1), 141, 2010. doi:10.1016/j.jtrangeo.2008.12.003.

Gutiérrez, J. and G. Gómez. The impact of orbital motorways on intra-metropolitan accessibility: the case of madrid's m-40. Journal of Transport Geography, 7(1), 1, 1999. doi:10.1016/ S0966-6923(98)00029-5.

Hansen, W. How accessibility shapes land use. Journal of the American Planning Association, 25(2), 73, 1959.

Kwan, M.-P. Space-time and integral measures of individual accessibility: A comparative analysis using a point-based framework. Geographical Analysis, 30(3), 191, 1998. doi: 10.1111/j.1538-4632.1998.tb00396.x.

Lefebvre, N. and M. Balmer. Fast shortest path computation in time-dependent traffic networks. In Proceedings of the Swiss Transport Research Conference (STRC). Monte Verita, CH, 2007. See www.strc.ch.

Liu, S. and X. Zhu. Accessibility analyst: an integrated GIS tool for accessibility analysis in urban transportation planning. Environment and Planning B: Planning and Design, 31, 105, 2004 .

Martín, J. C. and A. Reggiani. Recent methodological developments to measure spatial interaction: Synthetic accessibility indices applied to high-speed train investments. Transport Reviews, 27(5), 551, 2007. doi:10.1080/01441640701322610.

Miller, E., K. Nagel, H. Ševčíková, D. Socha, and P. Waddell. OPUS: An open platform for urban simulation. In 9th Conference on Computers in Urban planning and urban management (CUPUM). University College London, UK, 2005. See www.cupum.org.

Moeckel, R. Business Location Decisions and Urban Sprawl A Microsimulation of Business Relocation and Firmography. Ph.D. thesis, University of Dortmund, 2006.

Nicolai, T. W. and K. Nagel. Coupling transport and land-use: Investigating accessibility indicators for feedback from a travel to a land use model. In Latsis Symposium 2012 - 1st European Symposium on Quantitative Methods in Transportation Systems. Lausanne, Switzerland, 2012. Also VSP WP 12-16, see www.vsp.tu-berlin.de/publications. 
OPUS User Guide. The Open Platform for Urban Simulation and UrbanSim Version 4.3. University of California Berkeley and University of Washington, 2011.

Raney, B. and K. Nagel. An improved framework for large-scale multi-agent simulations of travel behaviour. In P. Rietveld, B. Jourquin, and K. Westin, eds., Towards better performing European Transportation Systems, pp. 305-347. Routledge, London, 2006.

Rieser, M. and K. Nagel. Network breakdown "at the edge of chaos" in multi-agent traffic simulations. European Journal of Physics, 63(3), 321, 2008. doi:10.1140/epjb/e2008-00153-6.

Schirmer, P. Options and constraints of a parcel based approach in 'UrbanSimE'. In Proceedings of the 10th Swiss Transport Research Conference (STRC). 2010. See www.strc.ch.

Schirmer, P., C. Zöllig, B. R. Bodenmann, and K. W. Axhausen. The Zurich case study of UrbanSim. In European Regional Science Association Conference. 2011.

Train, K. Discrete choice methods with simulation. Cambridge University Press, 2003.

Vandenbulcke, G., T. Steenberghen, and I. Thomas. Mapping accessibility in Belgium: a tool for land-use and transport planning? Journal of Transport Geography, 17(1), 39, 2009. doi: 10.1016/j.jtrangeo.2008.04.008.

Vrtic, M., P. Fröhlich, and K. Axhausen. Schweizerische Netzmodelle für Strassen- und Schienenverkehr. In T. Bieger, C. Lässer, and R. Maggi, eds., Jahrbuch 2002/03 Schweizerische Verkehrswirtschaft, pp. 119-140. Schweizer Verkehrswissenschaftliche Gesellschaft, St. Gallen, 2003.

Waddell, P. Urbansim: Modeling urban development for land use, transportation, and environmental planning. Journal of American planning Association, 68(3), 297 , 2002.

Zhu, X. and S. Liu. Analysis of the impact of the MRT system on accessibility in Singapore using an integrated GIS tool. Journal of Transport Geography, 12(2), 89, 2004. doi:10.1016/j. jtrangeo.2003.10.003. 